\title{
Dosimetric study between a single isocenter dynamic conformal arc therapy technique and Gamma Knife radiosurgery for multiple brain metastases treatment: impact of target volume geometrical characteristics
}

Michel Chea ${ }^{1 *} \mathbb{C}$, Karen Fezzani ${ }^{1}$, Julian Jacob ${ }^{1}$, Marguerite Cuttat ${ }^{2}$, Mathilde Croisé ${ }^{1}$, Jean-Marc Simon ${ }^{1}$, Loïc Feuvret ${ }^{1}$, Charles-Ambroise Valery², Philippe Maingon ${ }^{1}$, Mohamed-Amine Benadjaoud ${ }^{3}$ and Catherine Jenny ${ }^{1}$

\begin{abstract}
Purpose: To compare linac-based mono-isocentric radiosurgery with Brainlab Elements Multiple Brain Mets (MBM) SRS and the Gamma Knife using a specific statistical method and to analyze the dosimetric impact of the target volume geometric characteristics. A dose fall-off analysis allowed to evaluate the Gradient Index relevancy for the dose spillage characterization.
\end{abstract}

Material and methods: Treatments were planned on twenty patients with three to nine brain metastases with MBM 2.0 and GammaPlan 11.0. Ninety-five metastases ranging from 0.02 to 9.61 cc were included. Paddick Index (PI), Gradient Index (Gl), dose fall-off, volume of healthy brain receiving more than $12 \mathrm{~Gy}\left(\mathrm{~V}_{12 \mathrm{~Gy}}\right)$ and DVH were used for the plan comparison according to target volume, major axis diameter and Sphericity Index (SI). The multivariate regression approach allowed to analyze the impact of each geometric characteristic keeping all the others unchanged. A parallel study was led to evaluate the impact of the isodose line (IDL) prescription on the MBM plan quality.

Results: For mono-isocentric linac-based radiosurgery, the IDL around 70-75\% was the best compromise found. For both techniques, the $\mathrm{Gl}$ and the dose fall-off decreased with the target volume. In comparison, PI was slightly improved with MBM for targets $<1$ cc or SI > 0.78. GI was improved with GP for targets $<2.5$ cc. The $V_{12 G y}$ was higher with MBM for lesions $>0.4$ cc or $\mathrm{SI}<0.84$ and exceeded 10 cc for targets $>5$ cc against 6.5 cc with GP. The presence of OAR close to the PTV had no impact on the dose fall off values. The dose fall-off was higher for volumes $<3.8 \mathrm{cc}$ with GP which had the sharpest dose fall-off in the infero-superior direction up to $30 \% / \mathrm{mm}$. The mean beam-on time was 94 min with GP against 13 min with MBM.

Conclusions: The dose fall-off and the $\mathrm{V}_{12 \mathrm{~Gy}}$ were more relevant indicators than the $\mathrm{Gl}$ for the low dose spillage assessment. Both evaluated techniques have comparable plan qualities with a slightly improved selectivity with MBM

\footnotetext{
*Correspondence: michel.chea@aphp.fr

${ }^{1}$ Radiation Oncology Department, Pitié-Salpêtrière Hospital, AP-HP

Sorbonne University, 47-83 Boulevard de l'Hôpital, 75651 Paris Cedex 13,

France

Full list of author information is available at the end of the article
}

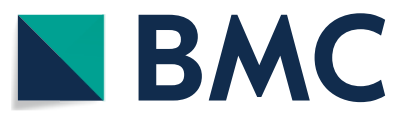

(c) The Author(s) 2021. Open Access This article is licensed under a Creative Commons Attribution 4.0 International License, which permits use, sharing, adaptation, distribution and reproduction in any medium or format, as long as you give appropriate credit to the original author(s) and the source, provide a link to the Creative Commons licence, and indicate if changes were made. The images or other third party material in this article are included in the article's Creative Commons licence, unless indicated otherwise in a credit line to the material. If material is not included in the article's Creative Commons licence and your intended use is not permitted by statutory regulation or exceeds the permitted use, you will need to obtain permission directly from the copyright holder. To view a copy of this licence, visit http://creativecommons.org/licenses/by/4.0/. The Creative Commons Public Domain Dedication waiver (http://creativeco mmons.org/publicdomain/zero/1.0/) applies to the data made available in this article, unless otherwise stated in a credit line to the data. 
for smaller lesions but with a healthy tissues sparing slightly favorable to GP at the expense of a considerably longer irradiation time. However, a higher healthy tissue exposure must be considered for large volumes in MBM plans.

Keywords: Stereotactic radiosurgery, Multiple brain metastases, Single-isocenter, Gammaknife, Gradient index, Dose fall-off, Target volume effect

\section{Introduction}

For the multiple brain metastases treatment, whole brain radiation therapy (WBRT) tends to disappear in favor of stereotactic radiosurgery (SRS) or stereotactic radiotherapy (SRT). One prospective study [1] indicated that there was no significant difference between two to four and five to ten metastases for the overall survival with SRS alone. No prospective studies have evaluated the use of SRS relative to whole brain radiotherapy (WBRT) for patients with more than four brain metastases. However, the current tendency is to avoid WBRT due to the attendant toxicity and neurological deterioration after such a treatment.

Historically, since 1968, Gammaknife radiosurgery is the gold standard for stereotactic treatments. The Gamma Knife (Elekta, Stockholm, Sweden) contains 192 ${ }^{60}$ Co sources and 4,8 and $16 \mathrm{~mm}$ collimator options providing 192 narrow beams precisely focused on a target.

Other SRS techniques have been developed since using linear accelerators. Examples include multiple isocenter dynamic conformal arc therapy (MIDCA) which traditionally assigns one group of non-coplanar dynamic conformal arcs to each target, volumetric modulated arc therapy (VMAT), and CyberKnife radiosurgery (Accuray, Sunnyvale, CA, USA).

In 2016, Elements Multiple Brain Mets SRS (MBM) version 1.5 (Brainlab, Munich, Germany) revived dynamic conformal arcs as the paradigm for linac-based stereotactic radiosurgery [2-4]. Automatic treatment planning can be performed on multiple metastases simultaneously with non-coplanar dynamic conformal arcs (DCA) sharing the same isocenter (barycenter targets).

Similarly, HyperArc VMAT (Varian, Palo Alto, CA, USA) can also offer single-isocenter treatments for multiple brain metastases and seems to be a promising solution as well.

Several publications have compared the available techniques for multiple brain metastases treatments. All solutions are capable of achieving a high level of conformity; differences are found for dose spillage, planner dependency and the beam on time. Manual VMAT is reported to have a high dose spillage, a dependency on planning skills and an inter-planner variability [2, 3, 5-7]. MBM version 1.5 had a slightly lower conformity reported in few articles $[5,8]$ but proved a plan quality similar to MIDCA for a shorter beam delivery time [3]. Since 2019, version 2.0 is commercially available with improvements in the optimization engine and a dosimetric study compared this approach with manual VMAT [6], in which the new algorithm in MBM 2.0 compared well to VMAT, even when using multiple isocenters (one plan per target) for the latter. The authors concluded that Elements showed better plan quality in terms of selectivity and dose spillage, as well as treatment time. Another dosimetric study for MBM 2.0 compared MBM version 1.5 and 2.0 with different MLC designs [9]: HDMLC (Varian, Palo Alto, CA, USA) and Agility MLC with and without jaw tracking feature (Elekta, Stockholm, Sweden). The authors demonstrated the added values of the version 2.0 and the potential incremental benefits of improved software optimization and MLC design. HyperArc combines high conformity and high dose gradient close to the Gamma Knife with more efficiency in terms of beam-on time. $[5,8,10,11]$. The Gamma Knife remains however a reference in terms of stereotactic treatments because of its well-known high dose gradient and high localization precision $[5,7]$ despite a long beam-on time and an invasive procedure to fix the mask on the skull.

All these studies were performed with a basic statistical analysis on targets of different geometrical characteristics inducing probably a bias in the comparison.

The dose spillage is often characterized by the Gradient Index but the dose fall-off is also a relevant parameter to analyze, useful in clinical conditions and considers the isodose direction. Only few articles have studied the dose fall-off; Munshi et al. [12] reported the dose fall-off pattern for the frameless SRS with 3D conformal Radiotherapy and VMAT. For both techniques, the sharpest dose fall-off was in the superior, inferior and OAR direction. It would be interesting and of a significant clinical help to have these kind of data for the other stereotactic techniques and correlate them to the widely used Gradient Index.

The authors propose a study to evaluate the improved actual version of MBM by comparison to the Gamma Knife SRS using a specific statistical method for a large number of metastases and to analyze the dosimetric impact of the target volume geometric characteristics. It also includes a dose fall-off analysis to determine the sharpest dose fall-off directions and to evaluate the Gradient Index relevancy for the dose spillage evaluation. 
Table 1 Characteristics of the lesions included in the study

\begin{tabular}{ll}
\hline Number of patients & $\mathbf{2 0}$ \\
\hline Number of metastases & 95 \\
Mean volume $(\mathrm{cc})$ & $0.76 \pm 1.35$ \\
Median volume $(\mathrm{cc})$ & 0.3 \\
Volume range $(\mathrm{cc})$ & 0.02 to 9.61 \\
Mean major axis diameter $(\mathrm{cm})$ & $1.17 \pm 0.65$ \\
Major axis diameter range $(\mathrm{cm})$ & 0.35 to 4 \\
Median major axis diameter $(\mathrm{cm})$ & 1 \\
\hline
\end{tabular}

\section{Material and methods \\ Patient selection}

Twenty patients previously treated in our Institution between 2018 and 2019 with Gammaknife SRS (Perfexion model) were selected. The patients had three to nine brain metastases from different primary malignant tumors: lung, breast kidney or melanoma. In total, ninety-five metastases with a major axis diameter ranging from 0.3 to $4 \mathrm{~cm}$ and a volume ranging from 0.02 to $9.61 \mathrm{cc}$ were included (Table 1). Ten of them were inside or close to the brainstem. No patient suffered from meningeal metastases.

\section{Treatment planning}

All patients had multimodality imaging with at least one magnetic resonance imaging (MRI) sequence dedicated for SRS (slice thickness $1.2 \mathrm{~mm}$ ) T1 FSPGR on the $1.5 \mathrm{~T}$ Optima 450 MR (GE Healthcare, Chicago, IL, USA) and one computed tomography (CT) scan dedicated protocol (slice thickness $1.25 \mathrm{~mm}$ ) on the Discovery $750 \mathrm{HD}$ (GE Healthcare, Chicago, IL, USA). These sequences benefited from a quality assurance, to ensure high quality of images and minimal distortion.

All the cases were replanned using GammaPlan (GP) version 11.0 and Multiple Brain Mets version 2.0.

In order to reduce inter-operator variabiliy, GP plans were recalculated by two physicists and MBM by another single physicist.

The Gross Target Volume (GTV) was delineated using the MRI T1 FSPGR sequence. The GTV was assigned as the PTV and thus, no margin was applied. The prescribed dose was 20 Gy on the reference isodose: $50 \%$ in GP, variable from 47 to $92 \%$ automatically selected by MBM. The dose was set at 12 Gy for cases where the volume of the brainstem exposed at a dose above $12 \mathrm{~Gy}$ was more than $0.5 \mathrm{cc}$ if 20 Gy was prescribed. The OAR constraints applied are reported in Table 2. [13-18]. Clinical constraints aimed to limit the volume of healthy brain receiving 12 Gy to $10 \mathrm{cc}$ in 1 fraction.
Table 2 Organs at risk constraints

\begin{tabular}{ll}
\hline Optic nerves & $\mathrm{D}_{\max }<10 \mathrm{~Gy}$ \\
Eyes & $\mathrm{D}_{\max }<10 \mathrm{~Gy}$ \\
Optic chiasm & $\mathrm{D}_{\max }<8 \mathrm{~Gy}$ \\
Brainstem & $\mathrm{V}_{12 \mathrm{~Gy}}<0.5 \mathrm{cc}$ \\
Normal brain & $\mathrm{V}_{12 \mathrm{~Gy}}<10 \mathrm{cc}$ \\
\hline
\end{tabular}

For MBM planning on the Novalis ${ }^{\circledR}$ Truebeam $^{\text {TM }}$ STx (Varian, Palo Alto, CA, USA) with $6 \mathrm{MV}$ photon beams (600 MU/min) and HD120 MLC, templates with number of table angles ranging from five to eight with two arc passes at each table angle were used depending on the spatial distribution of the metastases in the brain. The software automatically optimized the arc set-up geometry, MLC apertures, collimator angles and arc weighting with a couch angulation from $10^{\circ}$ to $320^{\circ}$ and collimator angles from $4^{\circ}$ to $45^{\circ}$. Metastases lining up in the direction of leaf-motion are automatically not treated simultaneously to restrict normal tissue exposure. Each case was customized: arc lengths were selected in order to avoid eyes and to prevent isodose from surrounding two neighboring metastases, optimization volumes were set either as an organ at risk (OAR) between two lesions or as PTV to increase the coverage and two isocenters were used if metastases were far from each other.

\section{Plan comparison}

For the plan comparison, several established dosimetric indices were used.

All the recalculated plans were accepted for a conformity index (CI) equal to 1 .

The CI [19] characterizes the coverage and is defined as:

$$
\mathrm{CI}=\frac{\mathrm{TV} \text { PIV }}{\mathrm{TV}}
$$

where $T V_{\mathrm{PIV}}$ is the volume of the target covered by the prescription isodose and TV is the target volume.

The selectivity is evaluated by the Paddick Index (PI) [20] which is defined as:

$$
\mathrm{PI}=\frac{\mathrm{TV}_{\mathrm{PIV}}^{2}}{\mathrm{TVxPIV}}
$$

where PIV is the prescription isodose volume.

A score of 1 corresponds to an ideal isodose conformity to the target volume.

The dose spillage was evaluated by the volume of healthy brain receiving more than $12 \mathrm{~Gy}, \mathrm{~V}_{12 \mathrm{~Gy}}$, the dose fall-off and the Gradient Index (GI), which is defined as: 


$$
\mathrm{GI}=\frac{\mathrm{PIV}_{50}}{\mathrm{PIV}}
$$

where PIV ${ }_{50}$ corresponds to the volume of the half prescription isodose. The GI should be as low as possible.

The dose fall-off was expressed in $\% / \mathrm{mm}$. To obtain these data, the distance between the prescribed isodose (20 Gy) and the 80\% (16 Gy)-60\% (12 Gy)-50\% (10 Gy) and 20\% (4 Gy) were reported and denominated as $\mathrm{R}_{100-80}, \mathrm{R}_{100-60}, \mathrm{R}_{100-50}$ and $\mathrm{R}_{100-20}$ respectively (Fig. 1). The corresponding dose fall-off is defined as:

$$
\text { Dosefalloff }=\frac{\% \text { of dose loss from isodose } 100 \text { to isodose } \mathrm{X}}{\mathrm{R}_{100-\mathrm{X}}}
$$

where $\mathrm{X}$ is the percentage of the prescribed isodose.

The dose fall-off values were collected for ten lesions close to the brainstem in the anterior, posterior, left, right superior and inferior directions.

The dose volume histograms were also compared to assess the OAR sparing.

The two techniques were compared using these dosimetric indices and considered several covariates which were tumor volume, major axis diameter, Sphericity Index and distance from isocenter. The Sphericity Index (SI) is defined as the ratio of the surface of a perfect sphere having the same volume as the target volume to the surface of the target volume and reflects the complexity of the shape [21].

$$
\mathrm{SI}=\frac{\text { A sphere }}{\text { A target volume }}
$$

A simple geometry getting closer to a sphere will have a Sphericity Index near 1 whereas a complex shape will decrease from 1.

This study must take into account a major clinical parameter: the duration of the treatment time. The most objective data is the beam on time since the total treatment time can variate according to the imaging verification processes associated to the therapists training. For the Gammaknife, the beam on time was rescaled to the beam on time by using new Cobalt sources reloading (3.313 Gy/min).

\section{Parallel study}

A parallel study was led to evaluate the dosimetric impact of the isodose line (IDL) prescription available on the version 2.0 of MBM.

In the SRS Prescription mode, with controlled inhomogeneity via isodose line (IDL) prescription, the twenty selected patients were planned with the IDL prescription value set from 50 to $90 \%$ in steps of $5 \%$.

For each plan, the mean Paddick conformity index and the mean gradient index were determined for each metastases in each plan. The mean value of the normal brain exposed to 12 Gy was determined for each plan.

\section{Statistical analysis}

To evaluate and compare the impact of metastases covariates (tumor volume, major axis diameter, SI and distance from isocenter) on dosimetric parameters (PI, GI and the volume of the normal brain receiving at least $12 \mathrm{~Gy}$ ) between the two techniques, a multivariate linear mixed effect regression was conducted with random effects at the metastasis level taking into account the repeated measure (a dosimetric parameter of a given metastasis is measured using the two techniques) and the patient level (clustered structure of the data since several lesions can be observed within the same patient). Due to the multivariate regression approach, the covariates effects are estimated jointly and the impact of each covariate is interpreted by keeping all the others unchanged.

The same modeling strategy was adopted to study the intra and inter technique impact of several predictors (presence of an OAR near the PTV, directions, metastasis

\section{Dose fall-off}

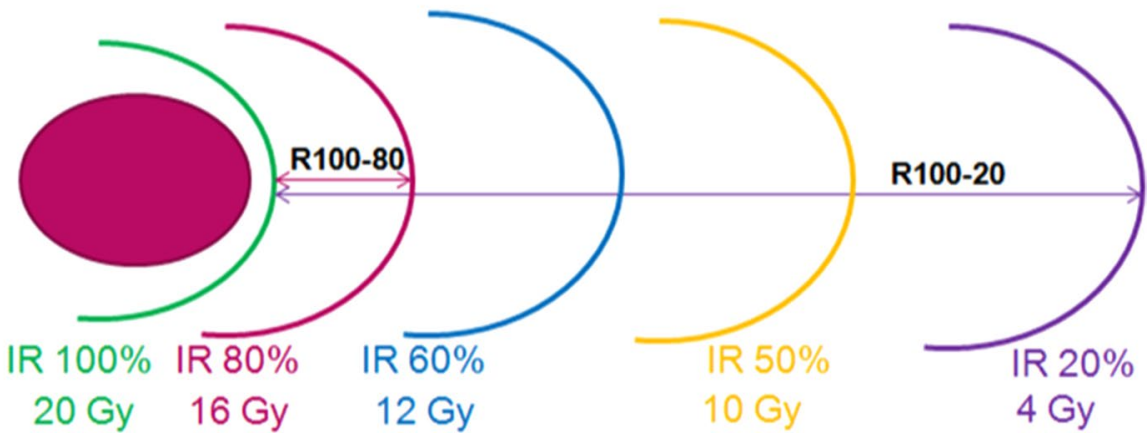

Fig. 1 Dose fall-off (R100-80, R100-60, R100-50, R100-20) reporting in mm 

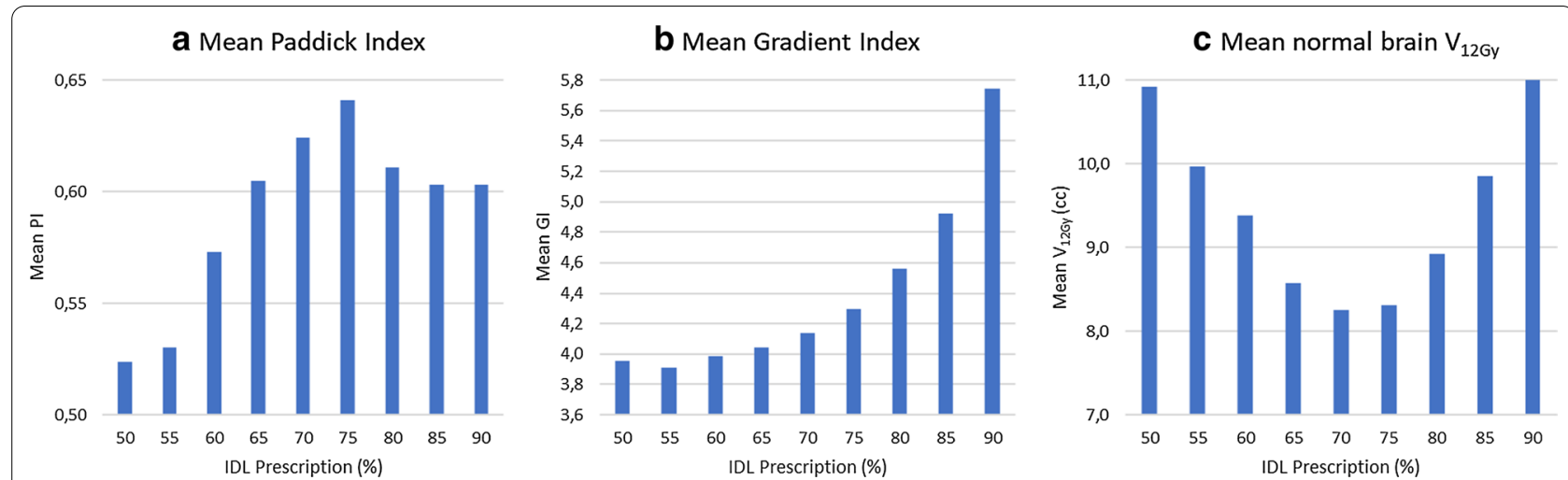

Fig. 2 Dosimetric impact of the isodose line prescription on MBM 2.0 concerning $\mathbf{a}$ the mean Paddick Index, $\mathbf{b}$ the mean Gradient Index and $\mathbf{c}$ the mean normal brain volume receiving at least 12 Gy

volume and isodoses range percentages) on dose fall-off values.

The OAR dose comparisons between the two techniques were performed within a functional data analysis framework: The Dx were defined as functions on the interval $[0,1]$ (range of $x$ percentages) and the inference on the difference between paired sample mean functions was conducted following Smaga et al. approach [22].

The reference here was Gamma Knife so results are expressed relative to this technique.

All analyses were performed using the MATLAB ${ }^{\circledR}$ software (version 8.2.0.701, R2013b, MathWorks@, Natick, Massachusetts).

\section{Results}

\section{Parallel study}

In the SRS prescription mode, the average PI increased from the IDL $50 \%$ to reach a maximum at the IDL $75 \%$ and then decreased. The extreme values found were 0.52 and 0.64 for the IDL $50 \%$ and $75 \%$ respectively (Fig. 2a). The gradient index increased with the IDL with a minimum value found for IDL 55\% and a maximum for IDL 90\% going from 3.91 to 5.74 (Fig. 2b) whereas the volume for the normal brain receiving at least 12 Gy decreased from IDL $50 \%$ with $10.92 \mathrm{cc}$ to reach a minimum value at $8.25 \mathrm{cc}$ for IDL $70 \%$ and after increased until $11.72 \mathrm{cc}$ for the IDL 90\% (Fig. 2c). In summary, the best compromise for selectivity and sparing tissues was around a 70-75\% IDL prescription.

\section{Plan comparison \\ Overall study}

Dosimetric results The mean and the near maximum $\left(D_{2 \%}\right)$ doses to the PTV were higher with Gamma Knife (Table 3). This difference was induced by the prescribed IDL, which is around $50 \%$ for GP and $73 \%$ for MBM. The
Table 3 Doses to PTV

\begin{tabular}{lll}
\hline $\mathbf{N}=\mathbf{9 4}$ & MBM & GP \\
\hline $\begin{array}{l}\text { Mean prescription } \\
\text { isodose (\%) }\end{array}$ & 73; range [47-92] & 50; range [45-60] \\
Dnear min (Gy) & $21.51 \pm 1.15$ & $21.56 \pm 3.33$ \\
Dmean (Gy) & $25.09 \pm 2.03$ & $30.31 \pm 2.79$ \\
Dnear max & $27.54 \pm 2.72$ & $38.34 \pm 2.84$ \\
\hline
\end{tabular}

Table 4 Dosimetric index results

\begin{tabular}{lllc}
\hline & MBM & GP & $\boldsymbol{p}$ value \\
\hline Mean PI & $0.57 \pm 0.12$ & $0.532 \pm 0.17$ & 0.07 \\
Mean Gl & $4.09 \pm 1.14$ & $3.22 \pm 0.55$ & $<0.001$ \\
MeanV $_{12 \mathrm{~Gy}}(\mathrm{CC})$ & $1.87 \pm 2.58$ & $1.70 \pm 2.32$ & 0.013 \\
\hline
\end{tabular}

IDL prescription automatically determined for MBM fitted well to the results of the parallel study to have the best compromise for selectivity and sparing tissues since it was between the 70 and 75\% IDL prescription. The near minimum doses were equivalent since we have assured the same PTV coverage for all the plans.

Paddick Index (PI) For all the lesions, taking all variables together, there was no statistically significant difference for the overall mean PI (Table 4). The PI increased with the target volume. The results were in favor of MBM for the smallest target volumes but an opposite trend was observed for PTV larger than $1 \mathrm{cc}$.

A further analysis with functional data illustrated the effects of volume, major axes and sphericity on the difference between the MBM and GP in terms of PI. 
In Fig. 3, the green segments of the abscissa axis highlight the range of values where the PI exhibits a significant difference between the two techniques.

With the target volume, the difference between the mean MBM PI and GP PI decreased from 0.05 to -0.05 . The mean MBM PI was significantly higher for smaller target volumes $(<1 \mathrm{cc})$. The trend reversed after $1.8 \mathrm{cc}$ without statistically significant differences.

With regard to the major axis diameter, the difference followed the same trend as with the volume varying from 0.1 to -0.1 and was equal to zero at $1.5 \mathrm{~cm}$. The results were statistically significant and in favor of MBM for small major axes $<1.3 \mathrm{~cm}$ and in favor of GP for diameter $>1.75 \mathrm{~cm}$.
Compared to the mean GP PI, the mean MBM PI increased with the sphericity to reach a maximum difference of 0.05 and is statistically significant for sphericity $>0.78$.

For the distance to the isocenter, the mean PI difference was constantly higher of 0.04 for MBM. A statistically difference was observed between 27 and $56 \mathrm{~mm}$ from the isocenter.

Gradient Index (GI) The overall mean GI was $4.09 \pm 1.14$ for MBM and $3.22 \pm 0.55$ for GP $(\mathrm{p}<0.001)$ (Table 4). The raw data presented in Fig. 4 demonstrates graphically that the GI decreased with increasing target volume and that
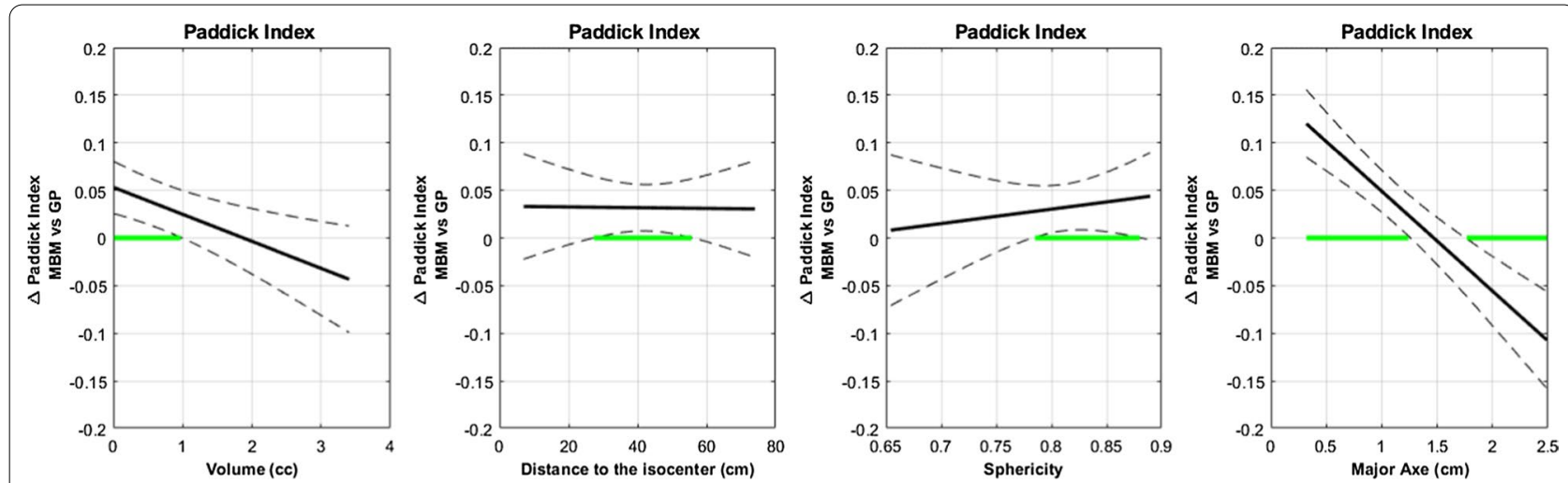

Fig. 3 Paddick Index absolute difference between MBM and GP as a function of the volume, the distance to the isocenter, the Sphericity Index and the major axis diameter determined with a multivariate regression approach. The green segments of the abscissa axis highlight the range of values where the difference is statistically significant

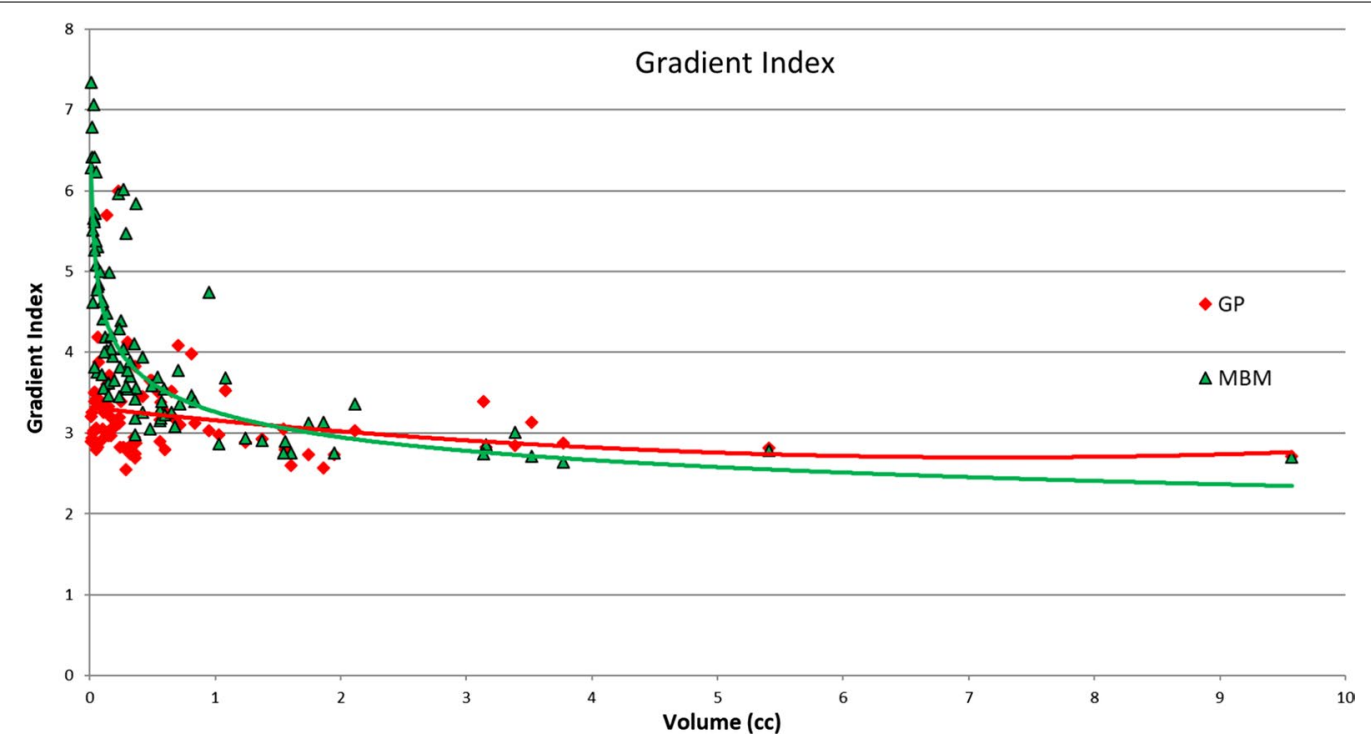

Fig. 4 Gradient Index as a function of the volume of the PTV 
the differences were mainly worse for MBM compared to GP for volumes smaller than $2 \mathrm{cc}$.

The functional data analyses also highlighted this trend (Fig. 5). The difference between the mean MBM GI and mean GP GI decreased from 1 to 0 with the target volume and was not statistically significant for volumes higher than $2.5 \mathrm{cc}$. Considering the major axis diameter this difference decreased from 1.8 to -0.5 and intersected the $\mathrm{x}$-axis for a major axis diameter equal to $2 \mathrm{~cm}$.
The mean MBM GI was significantly higher than the GP GI, irrespective of the sphericity index and the distance to isocenter considered (Fig. 5). With the sphericity index, the difference decreased from 1.1 to 0.8 whereas it increased from 0.6 to 1 with the distance to the isocenter.

Healthy brain tissue receiving at least 12 Gy The overall mean $\mathrm{V}_{12 \mathrm{~Gy}}$ was $1.87 \pm 2.58 \mathrm{cc}$ for $\mathrm{MBM}$ and $1.7 \pm 2.32$ for Gamma Knife (Table 4). The Fig. 6 represents the raw data of $\mathrm{V}_{12 \mathrm{~Gy}}$ as a function of the target volume; the cor-
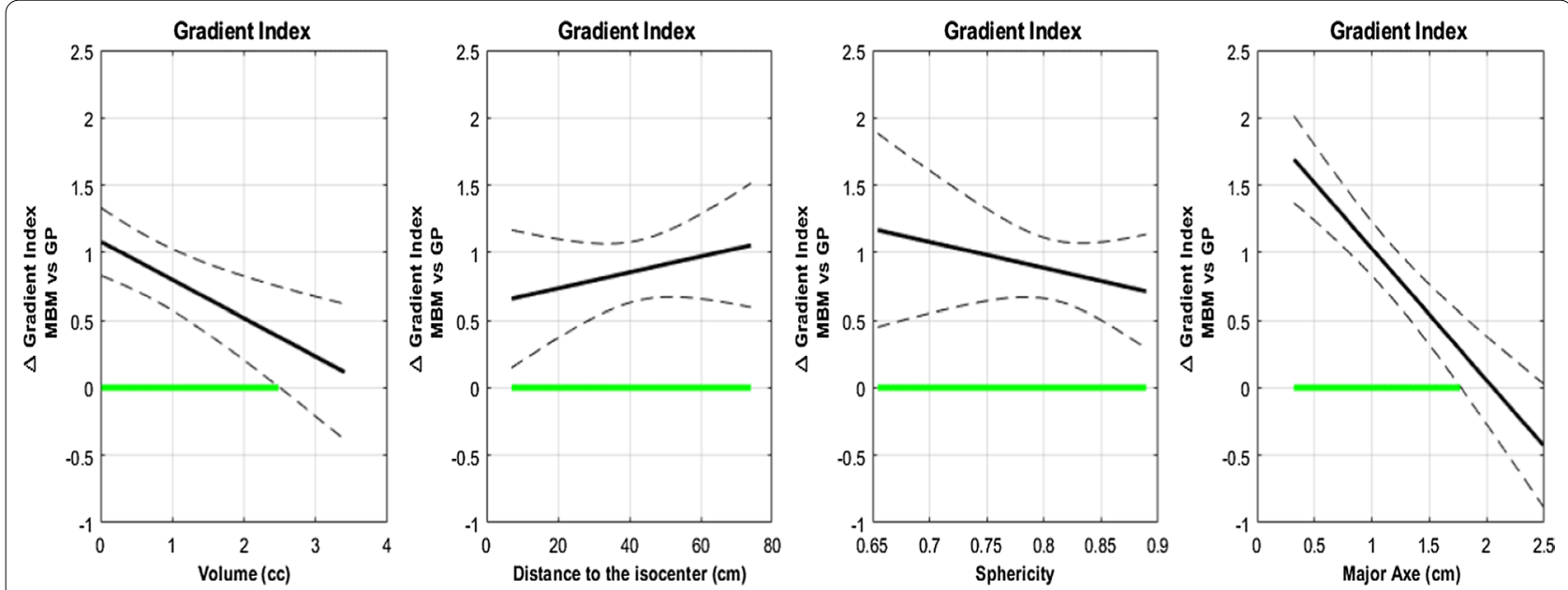

Fig. 5 Gradient Index absolute difference between MBM and GP as a function of the volume, the distance to the isocenter, the Sphericity Index and the major axis diameter determined with a multivariate regression approach

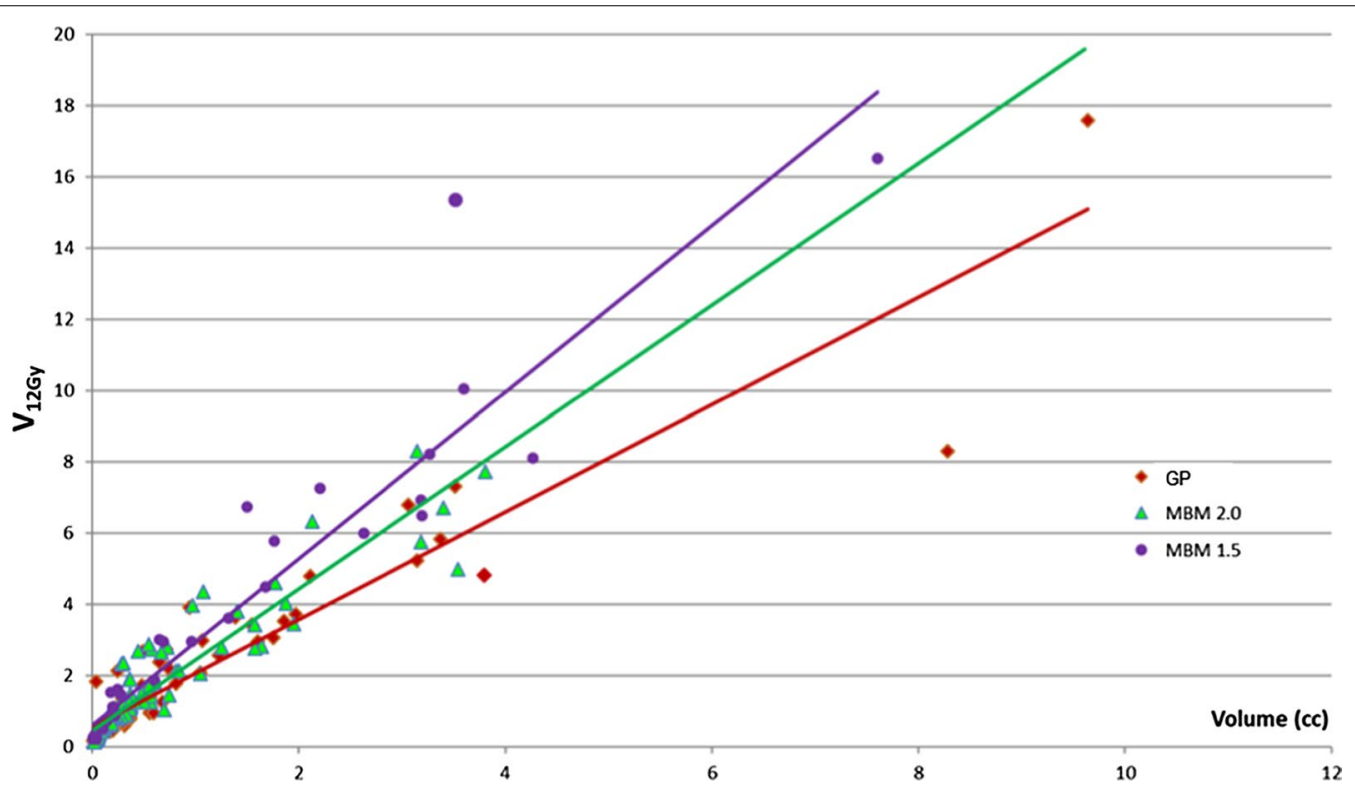

Fig. 6 Normal Brain volume receiving at least 12 Gy considering the planning target volume for GP and MBM 2.0. MBM 1.5 data from our previous study [29] are reported 
responding slope was 1.35 for Gamma Knife and 1.72 for MBM. This graph is clinically useful because it can determine approximatively the limitation of one kind of technique. The dose constraints regarding the healthy brain tissue were achieved for tumor volume below $5 \mathrm{cc}$ with MBM version 2.0, and $6.5 \mathrm{cc}$ for GP.

For the volume dependency, the difference between the mean MBM $V_{12 G y}$ and mean Gamma Knife $V_{12 G y}$ increased from 0 to $1 \mathrm{cc}$ and was statistically significant for volume higher than $0.4 \mathrm{cc}$ (Fig. 7).

With the major axis diameter, this difference increased from -0.4 to $1 \mathrm{cc}$ and intersected the $\mathrm{x}$-axis for a major axis diameter equal to $0.8 \mathrm{~cm}$ (Fig. 7).

With the sphericity index, the difference decreased from 0.6 to $0 \mathrm{cc}$ and was significant below a sphericity Index of 0.84 (Fig. 7).

According to the distance to the isocenter, the difference between the mean MBM $V_{12 G y}$ and the mean GP $\mathrm{V}_{12 \mathrm{~Gy}}$ increased from 0.15 to $0.25 \mathrm{cc}$ and was significantly higher for lesions located beyond $20 \mathrm{~cm}$ of the isocenter.

Organs at risk Each of the plans met the OAR and normal tissue dose constraints. The MBM OAR doses were superior to the Gamma Knife doses but the differences were not found to be clinically relevant. Considering the very low doses and the minor differences observed, the results of the eyes, optic nerves and chiasma were not presented.

For the brainstem (Fig. 8), for more than $10 \%$ of the organ volume, the difference between the mean MBM and GP doses was more than $0.5 \mathrm{~Gy}$. The analysis below $10 \%$ of the volume included doses superior to $12 \mathrm{~Gy}$, which corresponded to the aim to be reached in terms of maximum dose, the deviations were not statistically significant.

\section{Targets close to the brainstem}

Dosimetric indexes By contrast with the overall study, the difference of the mean PI focused on the ten metastases close to the brainstem was always negative at -0.1 with significance from 1 to $3.5 \mathrm{cc}$, a sphericity from 0.73 to 0.8 and a major axis diameter from 1.4 to 2.4 which means that the MBM PI was always worse in these specific cases. On the other hand, considering the whole cohort, MBM PI was improved for small metastasis with high sphericity in the overall study (Fig. 9). For complex cases, GP can perform plans with high selectivity but the time of planning and the treatment time were not considered in this focused study. The evolution of the GI and the $\mathrm{V}_{12 \mathrm{~Gy}}$ were the same in both studies (Fig. 9).

For the ten selected patients, the mean volume of brainstem exposed at a dose higher than 12 Gy was $0.34 \pm 0.38 \mathrm{cc}$ for MBM and $0.19 \pm 0.24 \mathrm{cc}$ for GP (Wilcoxon signed rank test $\mathrm{p}=0.02$ ).

Dose fall-off The Table 5 shows the dose fall-off expressed in millimeter. These raw data are interesting for knowing the distance necessary to decrease the dose from 20 Gy to an isodose line corresponding to an OAR constraint dose. The mean $\mathrm{R}_{100-60}$ refers to the distance from the isodose $20 \mathrm{~Gy}$ to $12 \mathrm{~Gy}$ useful for the brainstem toxicity; the mean distance was $2.2 \pm 1.1 \mathrm{~mm}$ for GP and $2.6 \pm 0.9 \mathrm{~mm}$ for MBM. The dose fall off was steeper in Gamma Knife plans, probably due to the larger number of beams.

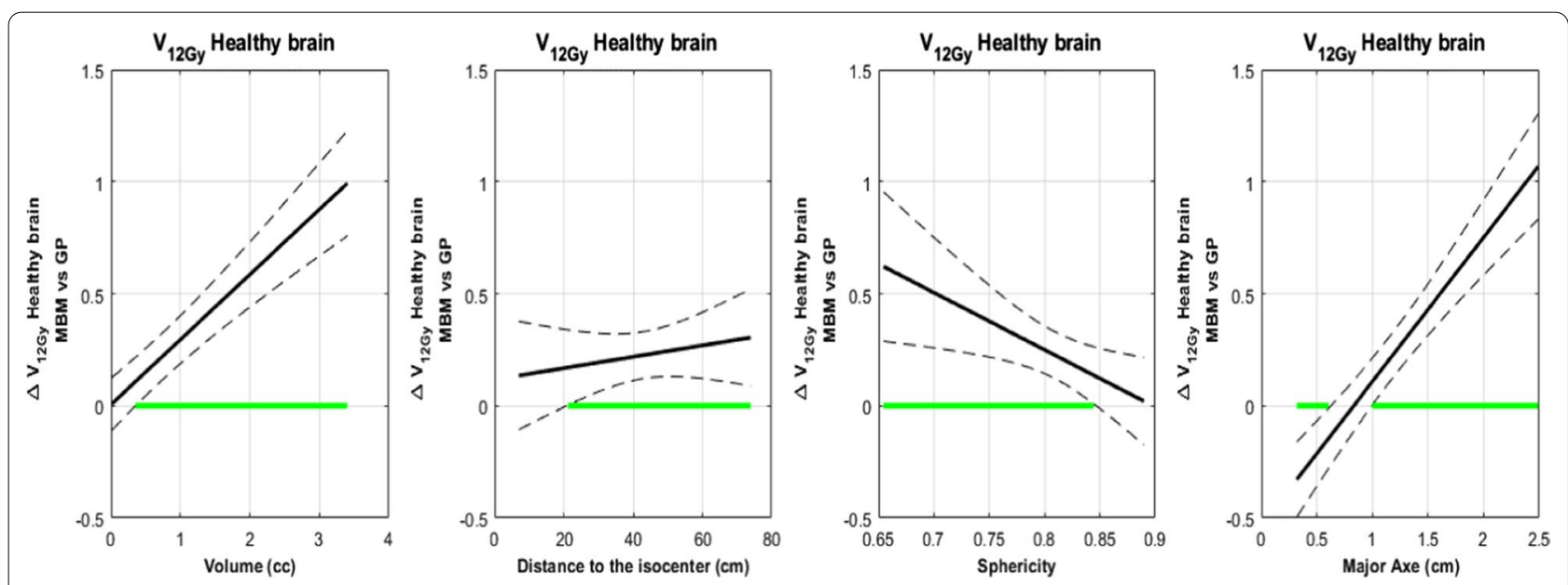

Fig. $7 V_{12 G y}$ absolute difference between MBM and GP according to the volume, the distance to the isocenter, the Sphericity Index and the major axis diameter determined with a multivariate regression approach 

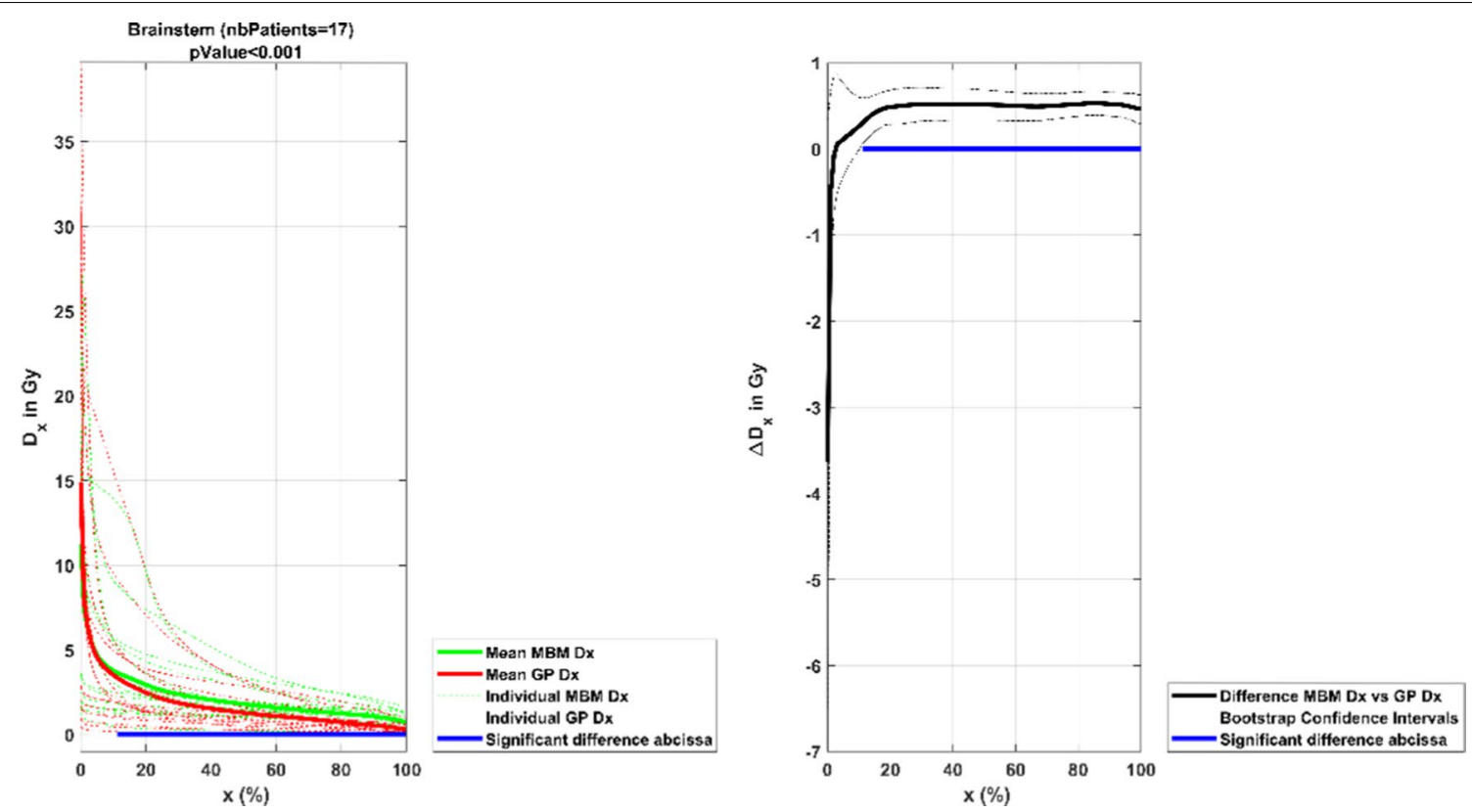

Fig. 8 Mean DVH comparison between the MBM and GP brainstem plotted in dose as a function of the volume. The graph on the left illustrates each individual DVH (dotted lines), the mean GP DVH (red continuous line) and mean MBM DVH (green continuous line). The graph on the right shows the mean absolute dose difference as a function of the volume. The blue line represents the range where the difference is statistically different

The dose fall-off expressed in \%/mm is the most appropriate definition and can give an order of magnitude of value comparable to other techniques already published.

The presence of an OAR close to the PTV had no impact on the dose fall-off: no improvement of the dose fall off value was observed neither with MBM nor GP. The dose fall off was even inferior on GP for lesions smaller than 2 cc (Fig. 10).

For both techniques, the mean dose fall off decreased with the volume and the isodose value (Fig. 11). For GP, it ranged from 5 to $30 \% / \mathrm{mm}$ whereas it ranged from 4 to $20 \% / \mathrm{mm}$ for MBM. The MBM dose fall-off was significantly lower for lesions smaller than $3.8 \mathrm{cc}$, this deviation increased when the volume and the isodose decreased to reach a maximum of $-12 \% / \mathrm{mm}$.

The GP dose fall-off had a preferential orientation (Fig. 12). In the inferior direction, the difference with the 4 other directions (anterior, posterior, right and left) decreased with the volume and was higher from 5 to $20 \% / \mathrm{mm}$. In the superior direction, the deviation with the 4 other directions was better of 6 to $20 \% / \mathrm{mm}$. The difference increased with the isodose and when the volume decreased.

For MBM, the effect of the direction was less evident (Fig. 13). For the inferior direction, the difference was significantly higher only for volume larger than $2 \mathrm{cc}$ and isodose $50 \%$ with a maximum difference at $5 \% / \mathrm{mm}$. For the superior direction, the difference with the 4 other directions increased with the volume and the isodose to reach a maximum at $6 \% / \mathrm{mm}$.

Treatment time All the GP plans were recalculated on $04 / 11 / 2019$, the mean beam on time was $169 \pm 47.5 \mathrm{~min}$ and rescaled to the date of the cobalt source reloading so the GK beam on time was divided by 1.8 and equal to $94 \pm 26.4 \mathrm{~min}$ (Wilcoxon signed rank test $p<0.001$ ). MBM beam on time was $13 \pm 3.2 \mathrm{~min}$ so the beam-on time is divided by 7.2 compared to the GP plans (Wilcoxon signed rank test $p<0.001)$.

\section{Discussions}

Despite two different approach of beam delivery (beam energy, number of isocenters) and treatment planning (manual or automatic), both techniques can achieve high quality treatment plans required for SRS. The impact of the target volume geometrical characteristics could have been assessed in isolation highlighting the importance of the volume effect.

Unlike GP, the use of MBM will obviously require margins applied to GTV to generate PTV mainly due to the delivery uncertainties of the linac and the positioning rotational residual errors. This consideration will probably affect the $\mathrm{V}_{12 \mathrm{~Gy}}$ and the OAR doses but to compare strictly the two techniques, the present study was 

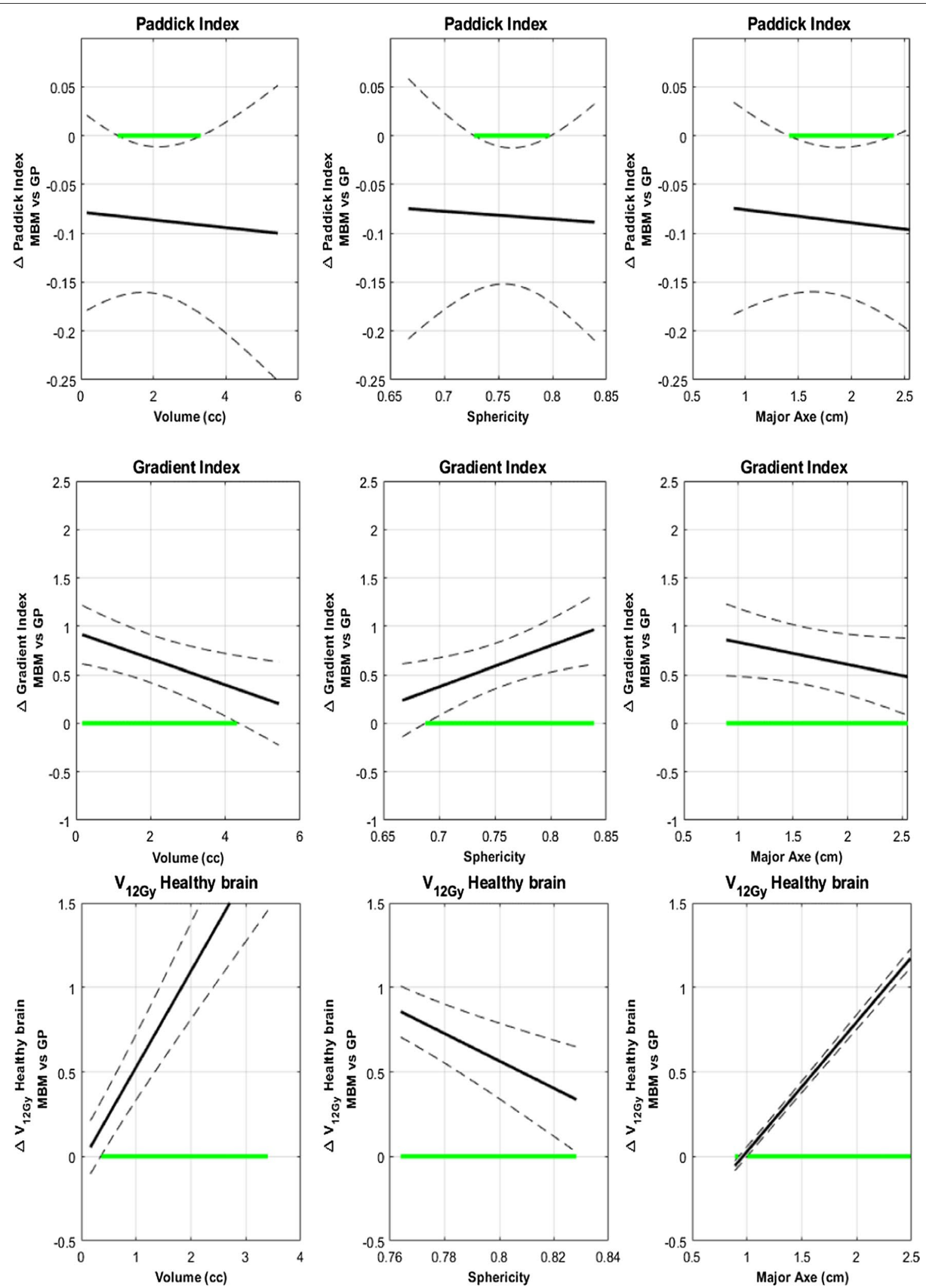

Fig. 9 Paddick Index, Gradient Index and $V_{12 G y}$ absolute difference between MBM and GP as a function of the volume, the distance to the isocenter, the Sphericity Index and the major axis diameter for the ten metastases close to the brainstem 
Table 5 Mean dose fall-off distance to decrease from the isodose prescription 100 to 80, 60, 50 and $20 \%$ towards the OAR direction or not for the targets close to the brainstem

\begin{tabular}{|c|c|c|c|c|c|c|c|c|}
\hline & \multicolumn{2}{|c|}{ Mean R 100-80 (mm) } & \multicolumn{2}{|c|}{ Mean R 100-60 (mm) } & \multicolumn{2}{|c|}{ Mean R 100-50 (mm) } & \multicolumn{2}{|c|}{ Mean R 100-20 (mm) } \\
\hline & GP & MBM & GP & MBM & GP & MBM & GP & MBM \\
\hline No OAR & $0.9 \pm 0.5$ & $1.2 \pm 0.3$ & $2.1 \pm 1.1$ & $2.6 \pm 0.9$ & $2,9 \pm 1.6$ & $3.6 \pm 1.4$ & $8.3 \pm 5.5$ & $12,3 \pm 7$ \\
\hline OAR & $0.9 \pm 0.4$ & $1.1 \pm 0.2$ & $2.3 \pm 1.2$ & $2.5 \pm 0.6$ & $3.3 \pm 1.7$ & $3.5 \pm 1.2$ & $9.7 \pm 5.3$ & $11.4 \pm 5.4$ \\
\hline Total & $0.9 \pm 0.5$ & $1.2 \pm 0.3$ & $2.2 \pm 1.1$ & $2.6 \pm 0.8$ & $3 \pm 1.6$ & $3.6 \pm 1.3$ & $8.6 \pm 5.4$ & $12 \pm 6.6$ \\
\hline
\end{tabular}
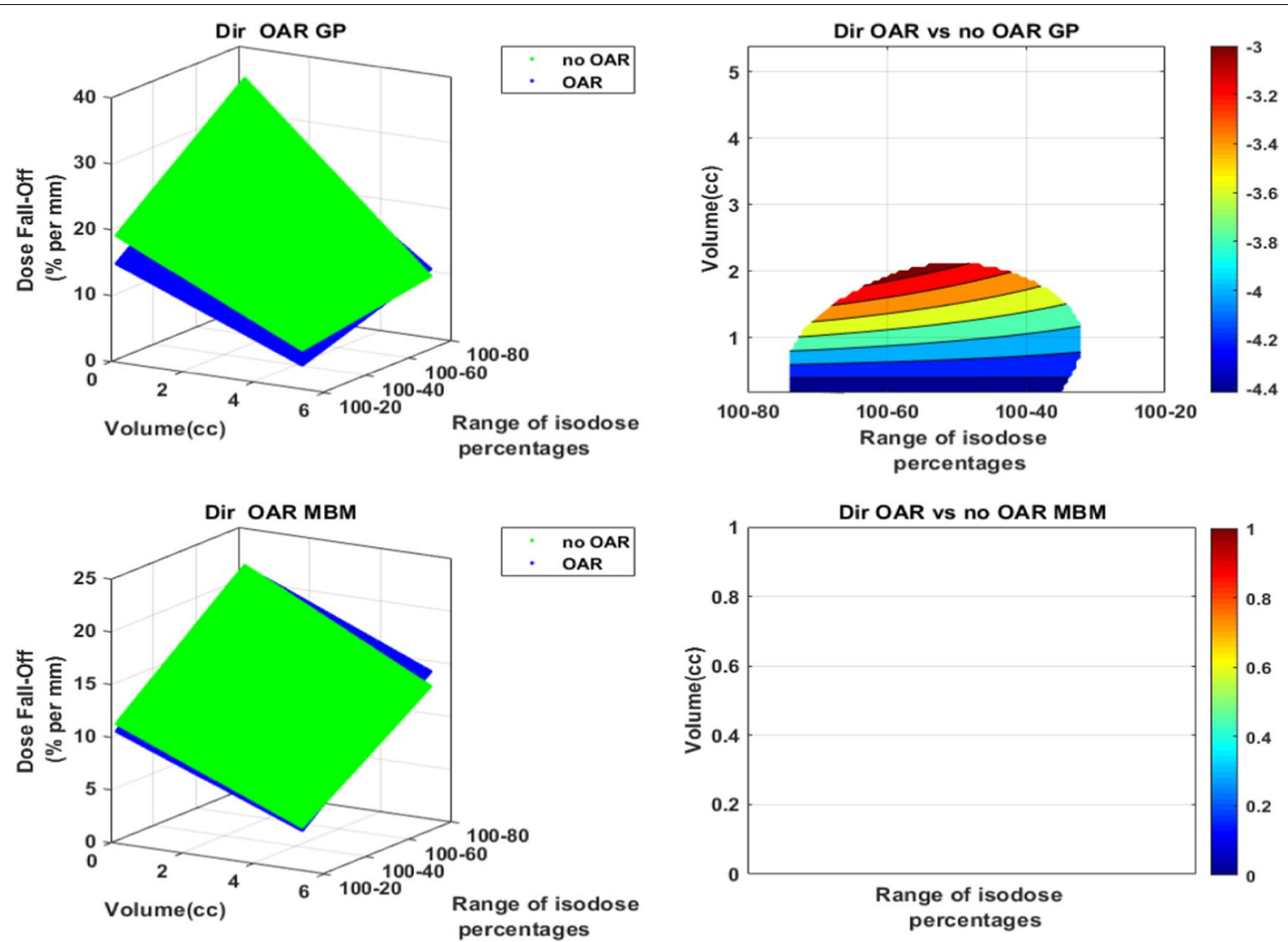

Fig. 10 Dose fall-off $(\% / \mathrm{mm})$ representation as a function of the volume and the isodose line in the OAR direction versus the "No OAR" direction. The figures on the left illustrate in 3D the dose fall-off absolute values as a function of the volume of the PTV and the isodose percentages selected. The graphs on the right indicate the absolute dose fall-off difference. The white areas are for differences not statistically significant

performed without margin. The margin used on a linac for a mono-isocentric technique can be of $0,1,2 \mathrm{~mm}$ or even non-uniform [23] depending on the distance from the isocenter and the repositionning tolerance. Considering the possibilities in GP, it's also possible to add margins so we cannot exclude that some groups use a margin on the GP.

Using a monoisocentric technique, any rotational residual errors will be amplified with subsequent offaxis localization errors and can be a concern to take into account with consequences in clinical practice. Over
$7-8 \mathrm{~cm}$ from isocenter, there is a need to add $1 \mathrm{~mm}$ margin $[24,25]$. Prentou et al. [26] evaluated the dosimetric impact of simulated rotational positional errors for multiple metastases VMAT SRS cranial cases. For single isocenter plans and $1^{\circ}$ rotation, the plan quality indices were significantly deteriorated for targets distant of more than $4 \mathrm{~cm}$ from the isocenter. For $2^{\circ}$ rotation, the conformity index was deteriorated by on average $7.2 \% / \mathrm{cm}$ and $2.6 \% /$ $\mathrm{cm}$ when two isocenters are used. Faught et al. [27] demonstrated that the linac mechanical uncertainties have also an impact on off axis targets with monoisocentric 
a

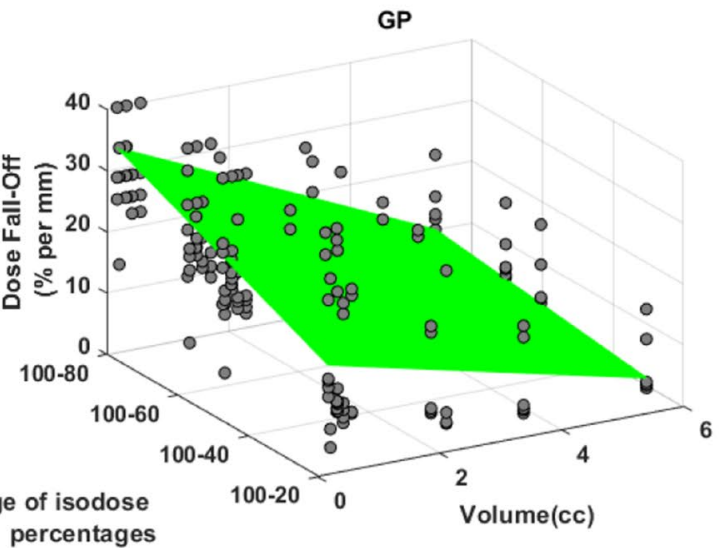

b

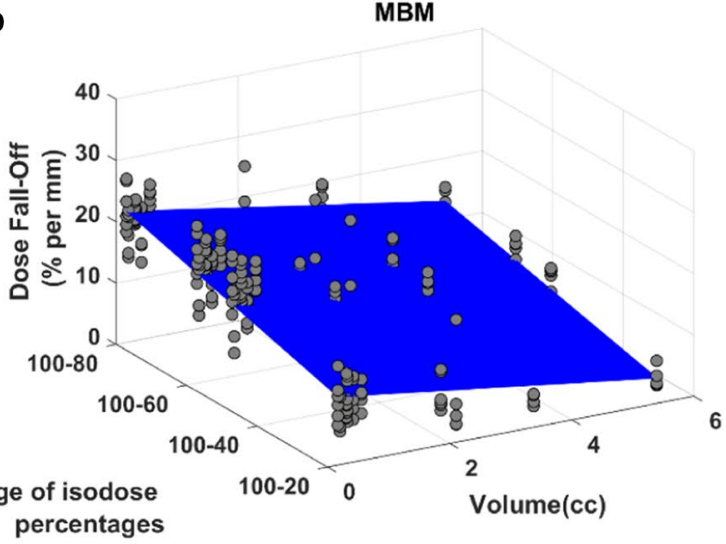

C

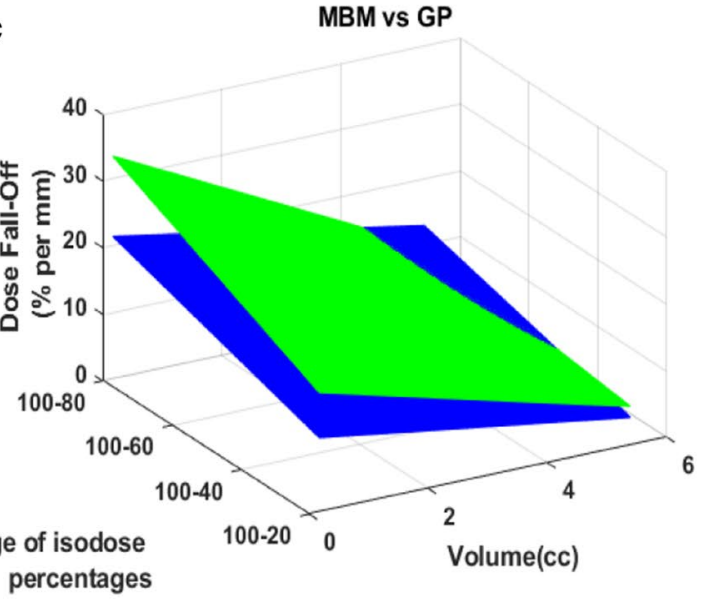

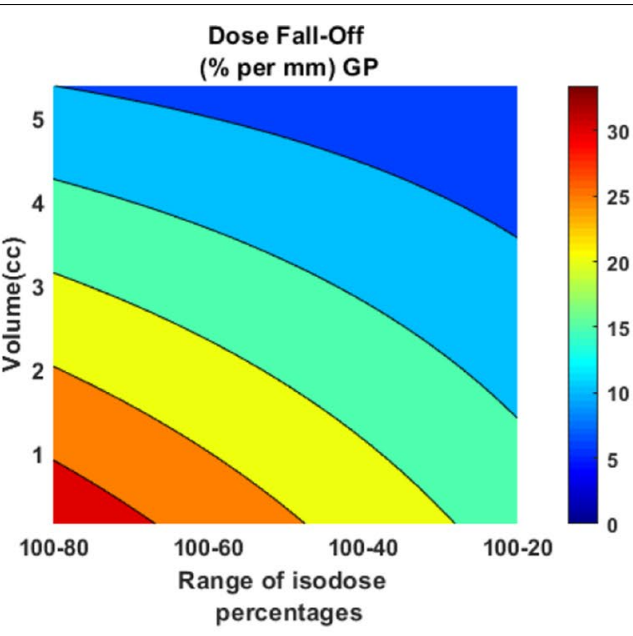

Dose Fall-Off

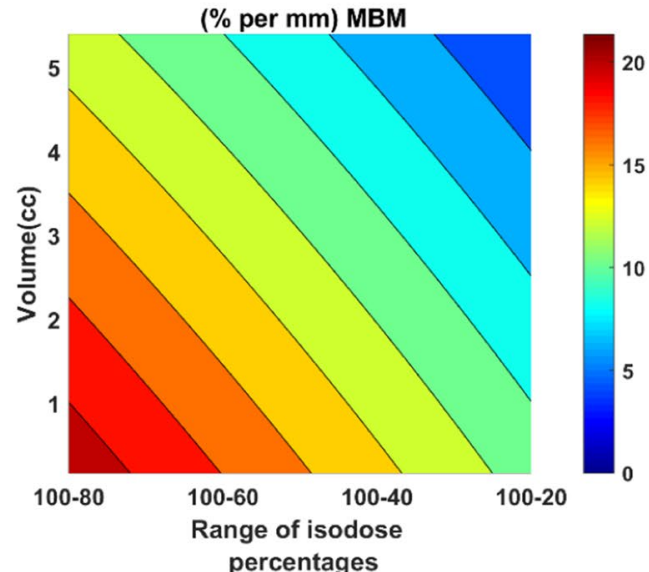

$\Delta$ Dose Fall-Off (\% per $\mathrm{mm})$ MBM vs GP

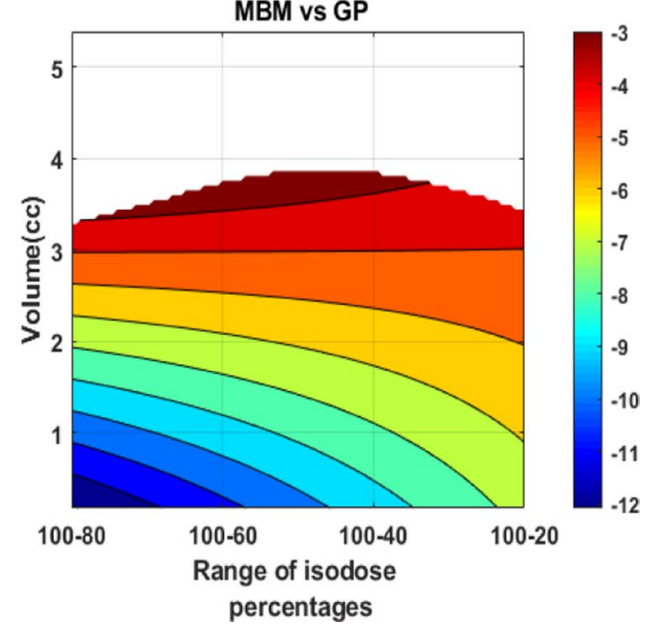

Fig. 11 Dose fall-off (\%/mm) representation as a function of the volume and the isodose line for GP (a) and MBM (b). Figure (c) illustrates the GP vs MBM dose fall-off: the left figure illustrate in 3D the dose fall-off absolute values as a function of the volume of the PTV and the isodose percentages selected, the right graph indicates the absolute dose fall-off difference. The white areas are for differences not statistically significant 

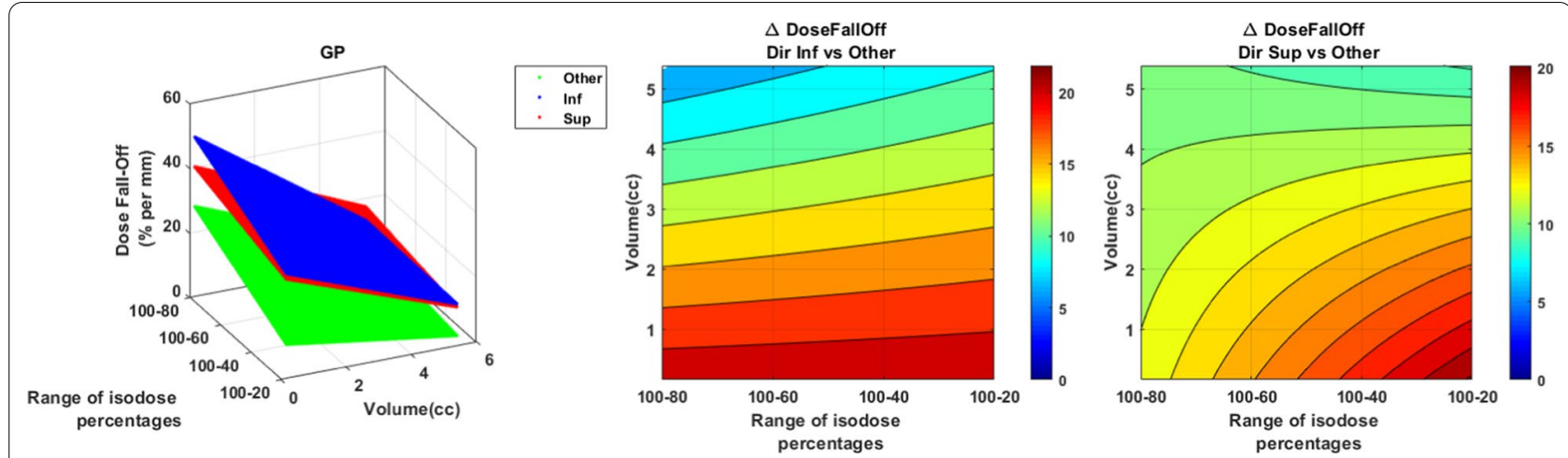

Fig. 12 GP dose fall-off $(\% / \mathrm{mm})$ representation as a function of the volume and the isodose line in the superior and inferior direction versus the "others" direction (anterior, posterior, left and right). The figure on the left illustrate in 3D the dose fall-off absolute values as a function of the volume of the PTV and the isodose percentages selected. The right graphs indicate the absolute dose fall-off difference

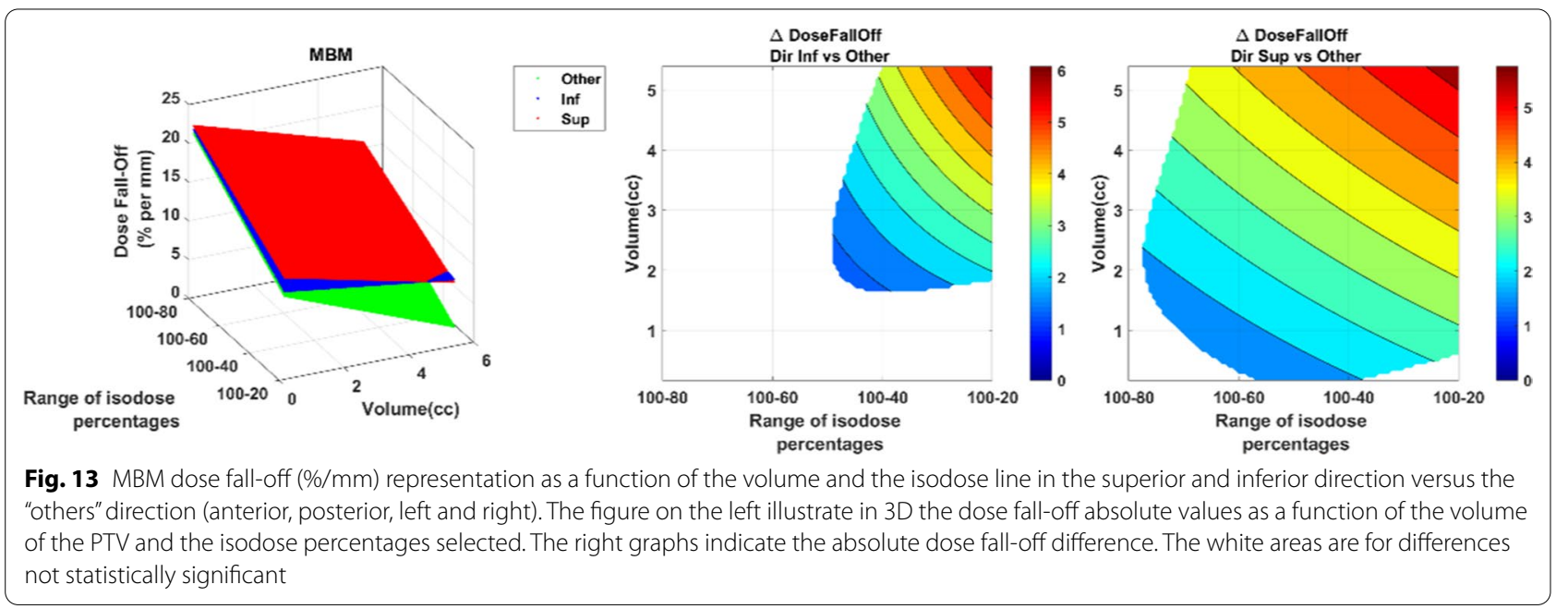

treatments. They studied the influence of induced errors on collimator, couch and gantry rotations at TG 142 tolerance levels. For multimet single isocenter VMAT plans, $1^{\circ}$ of collimator rotation led to an average change to $\mathrm{V} 100 \%$ and $\mathrm{D} 99 \%$ of $5 \%$ and $6 \%$ respectively. In the present study, only one case has required two isocenters because of two targets in extreme positions with a distance of $15 \mathrm{~cm}$ from each other. The treatment planning performed with one isocenter presented a poor selectivity. All these recommendations depend on the $4 \mathrm{D}$ or $6 \mathrm{D}$ correction, the repositioning tolerances and the mechanical tolerances and uncertainties of the linac.

Some publications showed the benefit of using a 6 MV FFF photon beam instead of a $6 \mathrm{MV}$ flattened beam in case of stereotactic treatments especially in terms of treatment time.

Viellevigne et al. [28] compared the use of a $6 \mathrm{MV}$ and a $6 \mathrm{MV}$ FFF for SBRT with DCA. They found a slight improvement of the conformity and the healthy tissue protection for a $6 \mathrm{MV}$ FFF beam especially for small volumes. With FFF beams, they had difficulties to cover and maintain a good homogeneity for large volumes because of the beam inhomogeneity and the MU were significantly increased. However the treatment time was considerably reduced due to the high dose rate (1400 MU/ min vs $600 \mathrm{MU} / \mathrm{min}$ ). Dzierma et al. [29] compared $7 \mathrm{MV}$ FFF to $6 \mathrm{MV}$ plans with quality indices, dose to OAR and out-of-field dose, in case of intracranial radiosurgery for multiple metastases with multiple non coplanar arcs. The 7 MV FFF plans were marginally superior to the flat beam $6 \mathrm{MV}$ plans with treatment times reduced almost by half. These studies were led with targets on the isocenter but due to the profile inhomogeneity, these conclusions can't be applied on a monoisocentric unmodulated technique. We didn't find any study showing the benefit of a $6 \mathrm{MV}$ FFF beam on the plan quality for such a technique. The 6 MV FFF beam was not available in our TPS to investigate this point in our study. The only clear advantage is 
the considerably reduced beam-on time, an important parameter for the intra-fraction motion management.

To our knowledge, two articles can be found currently for MBM version 2.0; one was published by Kuntz et al. They compared MBM version 2.0 to VMAT plans and they achieved very high conformity values with PI around 0.85 whereas our mean PI is $0.57 \pm 0.12$. The volume of the lesions considered in both studies can explain this difference. Kuntz et al. had mean GTV $+1 \mathrm{~mm}=2.3 \mathrm{cc}$ against $0.76 \mathrm{cc}$ in our study. In the present study, we observed that the volume of the target strongly influences the PI. Moreover, the PTV coverage objectives were not the same: $95 \%$ of the PTV must be covered by $\mathrm{D}_{98 \%}$ whereas we obtain $100 \%$ covered by $D_{100 \%}$ in our cases. The PTV volume differences could also explain the differences in terms of mean GI: $3.55 \pm 0.59$ in the article published by Kuntz et al. compared with $4.09 \pm 1.14$ reported in our work. The other dosimetric study available with MBM version 2.0 performed with HD120 MLC by Taylor et al. also achieved a better PI and GI than our study. The target volumes were also higher than our study (2.16 cc vs $0.76 \mathrm{cc}$ ) and could explain those differences.

More published data are available for MBM version 1.5. The mean absolute values of PI and GI are improved compared with our study's results but the mean volume of the lesions are still higher with different PTV coverage purposes [2, 5, 7, 8]. Narayanasami et al. [30] is the only study with PTV mean value close to those observed in our series: $0.7 \mathrm{vs} 0.76 \mathrm{cc}$ in our work. They found a PI 0.555 using MBM v1.5 which is comparable to our value of 0.57. Concerning the GK PI values obtained in our investigation, owing to the fact that we plan without margin, our clinical practice is to ensure the coverage to compensate the localization uncertainties.

The only dosimetric comparison between the Gammaknife and the MBM version 1.5 with enough statistics is the study led by Vergalasova et al. [5] MBM 1.5 had poorer PI and GI especially for sizes smaller than $1 \mathrm{~cm}$. We still obtain a worse GI for small lesions but PI is better for small targets in our study for the version 2.0.

Hofmaier et al. shows statistically significant correlations sphericity and $\mathrm{V}_{12 \mathrm{~Gy}}$ for MBM version 1.5 compared with VMAT plans and performs good healthy brain tissue sparing for high sphericity. In the present study, high sphericity is also favorable to MBM version 2.0 with a higher PI and a comparable $\mathrm{V}_{12 \mathrm{~Gy}}$ to GP.

Our previous work based on version 1.5 [31] showed equivalent selectivity but revealed difficulties to spare healthy tissues and OAR making the single isocenter dynamic conformal arc therapy (SIDCA) an interesting technique in terms of treatment time for patients with small lesions distant from each other. The latest version of MBM provides optimization algorithms to considerably reduce the healthy brain volume receiving more than 12 Gy and to cut $\mathrm{V}_{12 \mathrm{~Gy}}$ bridges between two brain metastases close to each other. A Normal Tissue Sparing (NT) slider in the graphical user interface can be used to control the dose gradient optimization. Increasing normal tissue sparing will typically decrease the beam's eye view margins around each target, increasing the heterogeneity inside the target and reducing the dose gradient outside the target. The $\mathrm{V}_{12 \mathrm{~Gy}}$ exceeded $10 \mathrm{cc}$ for PTV bigger than $2.5 \mathrm{cc}$ in the MBM 1.5 version compared with $5 \mathrm{cc}$ using the MBM 2.0 version. In the present study we were able to select patients with multiple lesions close to each other and to the brainstem. The study led by Taylor et al. also confirmed the plan quality improvements of the version 2.0 compared to the version 1.5 in terms of selectivity and dose spillage.

The arrangement of the HD MLC seems to have a low influence on the plan quality. In our study, the PI is constant despite larger leaves after $4 \mathrm{~cm}$ and the slightly GI increase slighly with the distance to isocenter. Stanhope et al. [32] showed a degradation of the quality plan (Conformity index and gradient index) for VMAT plans especially for small lesions with volume lower than $1 \mathrm{cc}$. Taylor et al. [9] also found an influence of the MLC design with a significant improvement of the plan quality. For the version 2.0 of MBM, the mean conformity index increased from 0.79 to 0.82 , the mean gradient index improved from 3.76 to 3.15 and the mean volume normal brain receiving more than 12 Gy decreased from 16.15 to $13.72 \mathrm{cc}$ when they switched from the Agility MLC of Elekta with leaves of $5 \mathrm{~mm}$ wide to the Varian 120 HDMLC with central leaves of $2.5 \mathrm{~mm}$ wide.

Figure 6, shows the volume of healthy brain receiving at least 12 Gy depending on the target volume but these results are a little bit overestimated because they are noted regardless of the number of lesions. Saghal et al. [33], found a significant increase in 12 Gy volume for multiple target treatments compared with single target treatments by approximately $4 \%$ per target when a high dose such as 20-24 Gy was used.

In our investigation, the PI only take into account PTV percentage covered by the prescription isodose. Dimitriadis et al. proposed the Efficiency Index to assess the treatment plan quality in SRS [34]. It combined conformity, gradient and mean dose into a single value and was adaptable to multiple target plans. This index is favorable to the GK because it takes into account the PTV mean dose and the $\mathrm{V}_{10 \mathrm{~Gy}}$ for the numerator and the denominator respectively.

Munshi et al. described a sharpest dose fall-off in the cranio-caudal direction in accordance with our results which indicates that this effect strongly depends on the treatment delivery because the 3D radiotherapy 
and the VMAT cases were performed with restricted couch angles. The preferential orientations can also be explained by the Gammaknife beam delivery method which is made up with 192 cobalt sources arranged in crown. The higher dose fall off found in regard of OAR is produced by the beam arrangement of the 3DRT and the ability of the VMAT to spare OAR. On the other hand, the techniques studied in our work seem to spare OAR and normal tissues by reducing the overall dose spillage with high heterogeneity inside the PTV and not by blocking one beam direction. The mean 3DRT/VMAT dose fall off $100-80,100-50$ and $100-20$ were $7.0 \pm 1.5,4.7 \pm 0.8$ and $4.1 \pm 1.6 \% / \mathrm{mm}$ respectively. These results are much lower compared to our data. The GK dose fall off 100$80,100-50$ and $100-20$ was $27.2 \pm 12.1,21.6 \pm 11.3$ and $14.4 \pm 10 \% / \mathrm{mm}$ and the MBM dose fall off $100-80$, $100-50$ and $100-2018.1 \pm 4.3,15.5 \pm 4.7$ and $8.5 \pm 3.9 \% /$ $\mathrm{mm}$. This can be explained by the considered PTV volume, Munshi et al. mean PTV volume was $11.7 \pm 16.1 \mathrm{cc}$ whereas our mean PTV volume was $1.47 \pm 1.75 \mathrm{cc}$. The Fig. 11 illustrates the strong decrease of the dose-fall off with the tumor volume.

In our study, none of the techniques shows a strong ability to protect OAR. For GP plans, there is a possibility to have strong gradient in one direction by plugging some shots but the treatment time is considerably increased and the isodose resulting, distorted. In our Institution, we plug the shots to protect small structures with high constraints like cochlea in acoustic schwannoma treatments. For our ten cases selected, the brainstem surrounds a large part of the metastasis making the plugging not really relevant.

The gradient index is commonly used to characterize the dose spillage but our study reveals the limit of this index as a good indicator of the quality plan for the dose fall-off and the $\mathrm{V}_{12 \mathrm{~Gy}}$ absolute value. In the dose fall-off analysis, compared to the Fig. 4, the results are conflicting because they both decrease with the volume. Moreover, the mean $\mathrm{V}_{12 \mathrm{~Gy}}$ deviation between GP and MBM increase with the volume (Fig. 6) whereas GI tends to become comparable with increasing volumes. This can be explained by the predominance of the PIV in the denominator part and his dependence to the selectivity [35]. In the parallel study, we expected that the Gradient Index and the $\mathrm{V}_{12 \mathrm{~Gy}}$ according to the IDL prescription would follow the same trend but the results are not correlated. This difference is counter-intuitive. Indeed the $\mathrm{V}_{12 \mathrm{~Gy}}$ is a global data which ponders the gradient by the selectivity. For small lesions treated with MBM, the selectivity is improved, the gradient is worse and $\mathrm{V}_{12 \mathrm{~Gy}}$ lower than using GP. For large metastases treated on GP, the selectivity is better, the gradient is equivalent and the $\mathrm{V}_{12 \mathrm{~Gy}}$ is lower than MBM. For high sphericity, with MBM, selectivity is higher, the gradient is worse and the $\mathrm{V}_{12 \mathrm{~Gy}}$ equivalent to the GP plans. So in this series, the $\mathrm{V}_{12 \mathrm{~Gy}}$ seems to rather be correlated to the selectivity. In summary, for the comparison, the Gradient Index does not reflect the dose spillage for two targets or two groups of targets of different volume. The comparison is relevant only if the lesions are equivalent in terms of volumes and selectivity.

\section{Conclusions}

For the mono-isocentric linac-based radiosurgery, the isodose line prescription around $70-75 \%$ was the best compromise between selectivity and sparing tissues. For both techniques, the selectivity and $\mathrm{V}_{12 \mathrm{~Gy}}$ increased with the target volumes while the gradient and dose fall-off decreased. In our series, the dose fall-off and the $\mathrm{V}_{12 \mathrm{~Gy}}$ were more relevant indicators than the Gradient Index for the low dose spillage assessment. Both evaluated techniques can achieve a high level of selectivity and dose fall-off, essential to the radiosurgery with preferred orientations according to the beam delivery technique. Overall, both produce comparable plan qualities with a slightly improved selectivity with MBM for smaller lesions but with healthy tissue and OARs sparing slightly favorable for GK at the expense of a considerably longer irradiation time. However, a higher healthy tissue exposure must be considered for large volumes in MBM plans.

\section{Abbreviations}

Cl: Conformity Index; CT: Computed tomography; CTV: Clinical target volume; DVH: Dose volume histogram; FFF: Flattening filter free; Gl: Gradient index; GP: Gamma Plan; GTV: Gross target volume; IDL: Isodose line; MBM: Multiple Brain Mets; MIDCA: Multiple isocenter dynamic conformal arcs; MLC: Multi leaf collimator; MU: Monitor unit; MRI: Magnetic resonance imaging; OAR: Organ at risk; PI: Paddick index; PTV: Planning target volume; SI: Sphericity index; SRS: Stereotactic radiosurgery; SRT: Stereotactic radiotherapy; VMAT: Volumetric modulated arc therapy; $\mathrm{V}_{12 \mathrm{~Gy}}$ : Healthy brain receiving at least $12 \mathrm{~Gy}$; WBRT: Whole brain radiotherapy.

\section{Acknowledgements}

None.

\section{Authors' contributions}

CJ and MC drafted the manuscript. CAV contoured the datasets. KF, CJ, MCU and $\mathrm{MC}$ performed the treatment planning. MB performed the data analysis. CJ and MC interpreted the data. JJ, MCr, PM and CJ critically revised the manuscript. All authors read and approved the final manuscript.

\section{Funding}

This work did not receive any specific funding.

\section{Availability of data and materials}

The datasets supporting the conclusions of this article are included within the article.

Ethics approval and consent to participate Not applicable.

\section{Consent for publication}

Not applicable. 


\section{Competing interests}

The authors were involved in a clinical cooperation of 6 months with Brainlab AG to benefit from the loan of the Multiple Brain Mets Software version 2.0 during this period.

\section{Author details}

${ }^{1}$ Radiation Oncology Department, Pitié-Salpêtrière Hospital, AP-HP Sorbonne University, 47-83 Boulevard de l'Hôpital, 75651 Paris Cedex 13, France. ${ }^{2}$ Neurosurgery Department, Pitié-Salpêtrière Hospital, AP-HP Sorbonne University, Paris, France. ${ }^{3}$ PSE-SANTE/SERAMED, Radiation Protection and Nuclear Safety Institute, Fontenay aux Roses, France.

Received: 1 December 2020 Accepted: 11 February 2021

Published online: 27 February 2021

\section{References}

1. Yamamoto M, Serizawa T, Shuto T, Akabane A, Higuchi Y, Kawagishi J, et al. Stereotactic radiosurgery for patients with multiple brain metastases (JLGK0901): a multi-institutional prospective observational study. Lancet Oncol. 2014;15(4):387-95.

2. Gevaert T, Steenbeke F, Pellegri L, Engels B, Christian N, Hoornaert MT, et al. Evaluation of a dedicated brain metastases treatment planning optimization for radiosurgery: a new treatment paradigm? Radiat Oncol. 2016;11:13.

3. Huang Y, Chin K, Robbins JR, Kim J, Li H, Amro H, et al. Radiosurgery of multiple brain metastases with single-isocenter dynamic conformal arcs (SIDCA). Radiother Oncol. 2014;112(1):128-32.

4. Mori Y, Kaneda N, Hagiwara M, Ishiguchi T. Dosimetric study of automaticbrain metastases planning in comparison with conventional multi-isocenter dynamic conformal arc therapy and gamma knife radiosurgery for multiple brain metastases. Cureus. 2016;8(11):e882

5. Vergalasova I, Liu H, Alonso-Basanta M, Dong L, Li J, Nie K, et al. Multi-institutional dosimetric evaluation of modern day stereotactic radiosurgery (SRS) treatment options for multiple brain metastases. Front Oncol. 2019:9:483.

6. Kuntz L, Matthis R, Wegner N, Lutz S. Dosimetric comparison of monoisocentric and multi-isocentric plans for oligobrain metastases: a single institutional experience. Cancer Radiother. 2020;24(1):53-9.

7. Liu H, Andrews DW, Evans JJ, Werner-Wasik M, Yu Y, Dicker AP, et al. Plan quality and treatment efficiency for radiosurgery to multiple brain metastases: non-coplanar rapidarc vs. gamma knife. Front Oncol. 2016;6:26.

8. Ruggieri R, Naccarato S, Mazzola R, Ricchetti F, Corradini S, Fiorentino A, et al. Linac-based radiosurgery for multiple brain metastases: comparison between two mono-isocenter techniques with multiple non-coplanar arcs. Radiother Oncol. 2019;132:70-8.

9. Taylor M, Williams J, Gleason JF Jr. Effects of multileaf collimator design and function when using an optimized dynamic conformal arc approach for stereotactic radiosurgery treatment of multiple brain metastases with a single isocenter: a planning study. Cureus. 2020;12(8):e9833.

10. Ruggieri R, Naccarato S, Mazzola R, Ricchetti F, Corradini S, Fiorentino A, et al. Linac-based VMAT radiosurgery for multiple brain lesions: comparison between a conventional multi-isocenter approach and a new dedicated mono-isocenter technique. Radiat Oncol. 2018;13(1):38.

11. Ohira S, Ueda Y, Akino Y, Hashimoto M, Masaoka A, Hirata T, et al. HyperArc VMAT planning for single and multiple brain metastases stereotactic radiosurgery: a new treatment planning approach. Radiat Oncol. 2018;13(1):13.

12. Munshi A, Sarkar B, Roy S, Ganesh T, Mohanti BK. Dose fall-off patterns with volumetric modulated arc therapy and three-dimensional conformal radiotherapy including the "organ at risk" effect. Experience of linear acceleratorbased frameless radiosurgery from a single institution. Cancer Radiother. 2019:23(2):138-46.

13. Noël G, Antoni D, Barillot I, Chauvet B. Delineation of organs at risk and dose constraint. Cancer Radiother. 2016;20(Suppl):S36-60.

14. Delannes M, Latorzeff I, Chand ME, Huchet A, Dupin C, Colin P. Radiotherapy of benign intracranial tumors. Cancer Radiother. 2016;20(Suppl):S88-95.

15. Hanna GG, Murray L, Patel R, Jain S, Aitken KL, Franks KN, et al. UK consensus on normal tissue dose constraints for stereotactic radiotherapy. Clin Oncol (R Coll Radiol). 2018;30(1):5-14.
16. Milano MT, Usuki KY, Walter KA, Clark D, Schell MC. Stereotactic radiosurgery and hypofractionated stereotactic radiotherapy: normal tissue dose constraints of the central nervous system. Cancer Treat Rev. 2011;37(7):567-78.

17. Milano MT, Grimm J, Soltys SG, Yorke E, Moiseenko V, Tomé WA, et al. Singleand multi-fraction stereotactic radiosurgery dose tolerances of the optic pathways. Int J Radiat Oncol Biol Phys. 2018;S0360-3016(18):30125-31.

18. Milano MT, Grimm J, Niemierko A, Soltys SG, Moiseenko V, Redmond KJ, et al. Single- and multifraction stereotactic radiosurgery dose/volume tolerances of the brain. Int J Radiat Oncol Biol Phys. 2020;50360-3016(20):34101-8

19. Feuvret L, Noël G, Mazeron JJ, Bey P. Conformity index: a review. Int J Radiat Oncol Biol Phys. 2006;64(2):333-42

20. Paddick I. A simple scoring ratio to index the conformity of radiosurgical treatment plans. Technical note. J Neurosurg. 2000;93(Suppl 3):219-22.

21. Hofmaier J, Bodensohn R, Garny S, Hadi I, Fleischmann DF, Eder M, et al. Single isocenter stereotactic radiosurgery for patients with multiple brain metastases: dosimetric comparison of VMAT and a dedicated DCAT planning tool. Radiat Oncol. 2019;14(1):103.

22. Smaga $L$. A note on repeated measures analysis for functional data. Adv Stat Anal. 2020;104:117-39.

23. Miao J, Xu Y, Tian Y, Liu Z, Dai J. A study of nonuniform CTV to PTV margin expansion incorporating both rotational and translational uncertainties. J Appl Clin Med Phys. 2019;20(12):78-86.

24. Winey B, Bussiere M. Geometric and dosimetric uncertainties in intracranial stereotatctic treatments for multiple nonisocentric lesions. J Appl Clin Med Phys. 2014;15(3):122-32.

25. Ezzell GA. The spatial accuracy of two frameless, linear accelerator-based systems for single-isocenter, multitarget cranial radiosurgery. J Appl Clin Med Phys. 2017;18(2):37-43.

26. Prentou G, Pappas EP, Logothetis A, Koutsouveli E, Pantelis E, Papagiannis P, et al. Dosimetric impact of rotational errors on the quality of VMAT-SRS for multiple brain metastases: comparison between single- and two-isocenter treatment planning techniques. J Appl Clin Med Phys. 2020;21(3):32-44.

27. Faught AM, Trager M, Yin FF, Kirkpatrick J, Adamson J. Re-examining TG-142 recommendations in light of modern techniques for linear accelerator based radiosurgery. Med Phys. 2016;43(10):5437.

28. Vieillevigne L, Bessieres S, Ouali M, Lanaspeze C. Dosimetric comparison of flattened and unflattened beams for stereotactic body radiation therapy: impact of the size of the PTV on dynamic conformal arc and volumetric modulated arc therapy. Phys Med. 2016;32(11):1405-14.

29. Dzierma Y, Nuesken FG, Palm J, Licht NP, Ruebe C. Planning study and dose measurements of intracranial stereotactic radiation surgery with a flattening filter-free linac. Pract Radiat Oncol. 2014;4(2):e109-16.

30. Narayanasamy G, Stathakis S, Gutierrez AN, Pappas E, Crownover R, Floyd JR, et al. A systematic analysis of 2 monoisocentric techniques for the treatment of multiple brain metastases. Technol Cancer Res Treat. 2017;16(5):639-44.

31. Chea M, Verrecchia E, Jacob J, Troussier I, Feuvret L, Borius PY, et al. Arcthérapie conformationnelle mono-isocentrique des métastases cérébrales multiples: planification automatisée et comparaison dosimétrique avec le Gammaknife ${ }^{\circledR}$ Perfexion ${ }^{\text {TM }}$. Cancer/Radiothérapie. 2017;21:685-6.

32. Stanhope C, Chang Z, Wang Z, Yin FF, Kim G, Salama JK, et al. Physics considerations for single-isocenter, volumetric modulated arc radiosurgery for treatment of multiple intracranial targets. Pract Radiat Oncol. 2016;6(3):207-13.

33. Sahgal A, Barani IJ, Novotny J Jr, Zhang B, Petti P, Larson DA, et al. Prescription dose guideline based on physical criterion for multiple metastatic brain tumors treated with stereotactic radiosurgery. Int J Radiat Oncol Biol Phys. 2010;78(2):605-8

34. Dimitriadis A, Paddick I. A novel index for assessing treatment plan quality in stereotactic radiosurgery. J Neurosurg. 2018;129(Suppl1):118-24.

35. Paddick I, Lippitz B, Paddick I, et al. A simple dose gradient measurement tool to complement the conformity index. J Neurosurg. 2006;105(Suppl):194-201.

\section{Publisher's Note}

Springer Nature remains neutral with regard to jurisdictional claims in published maps and institutional affiliations. 